\section{P11 (continued)}

Evaluation: Community Service Attitudes Scale (CSAS) was used to collect data. This CSAS scale quantified four phases: perception, moral obligation, reassessment and helping measures with sub-scales for each phase. In addition, the intervention group answered four open-ended questions including barriers and benefits of service learning. Students filled-out the survey at the beginning and end of semester. Data were analyzed using chi-square test and ANOVA. Over $85 \%$ of the students from the control and intervention classes reported participating in unpaid community service at least once a year. Both groups reported very positive attitudes except the attitude toward costs and no significance differences were found between intervention and control groups. Most common benefits service-learning students' mentioned were "feeling of accomplishment" and "seeing community issues up close" and barrier was "limited time."

Conclusions and Implications: Exposure to community service improved the attitudes. Service-learning faculty members must help students see that costs are outweighed by the benefits derived.

Funding: None.

\section{P12 Attitudes of University Dining Hall Patrons Toward Recycling-An Exploratory Study \\ Krisha Thiagarajah,PhD, RD, kthiagar@indiana.edu, Indiana University, SPH Building 116, 1025 E. 7th Street, Bloomington, IN 47405; K. Thompson, BS; V. Getty, RD, MEd}

Objective: Recycling materials properly reduces waste. However, successful recycling programs depend on environmentally responsible behavior. A survey was conducted to gain a better understanding of the attitudes and behaviors of patrons of a Midwestern university dining hall toward recycling.

Design, Setting and Participants: A 19-item questionnaire was used to collect information from the patrons. The questions include four items related to demographic characteristics of the participants, ten items addressing attitudes toward recycling, three items about recycling awareness, and two items on trash disposal behavior. The data were collected from patrons as they exited the dining hall. Eighty participants (33 male, 47 female) completed the survey for a $45 \%$ response rate. Most respondents were freshman $(56.3 \%)$, plus $22.5 \%$ sophomores and $20.0 \%$ juniors.

Outcome Measures and Analysis: Descriptive statistics were calculated and chi-square tests were used to analyze the data.

Results: Most respondents gave a positive response to all ten questions about attitude toward recycling. Gender showed no significant impact on attitude. Almost 98\% of the respondents agreed that recycling has many positive effects on the environment, $2 \%$ were neutral, and none disagreed. Seventy percent of respondents reported that they learned about recycling through classes, 25\% through news media, and 11\% from social media. Over $62 \%$ of patrons reported leaving the trash in the accumulator for sorting by the dishroom employees while the rest self-sorted. Only $47.5 \%$ reported recycling consistently.

Conclusions and Implications: Dining hall patrons consistently reported pro-recycling attitudes. Yet less than half reported consistently recycling. Further studies should investigate how to convert positive attitudes toward recycling into actual recycling behavior among college students.

Funding: None.

\section{P13 An Online Introductory Nutrition Course Adapted for Hawai'i and the Pacific}

M. Kainoa Fialkowski, PhD, RD, LDN, mariekf@hawaii.edu, University of Hawaii at Manoa, 1955 East West Road, AgSci 216, Honolulu, HI 96822; W. Gibson, BS; E. Yiu; J. Banna, PhD, RD; G. Lin, PhD; M. Stewart, PhD; R. Novotny, PhD, RD, LDN

Objective: To gather student feedback on an online introductory nutrition course (OINC) adapted for Hawai'i and the Pacific (HP) region.

Target Audience: Students in an OINC-HP at the University of Hawai'i (UH) at Mānoa $(n=71)$ in fall 2014.

Theory, Prior Research, Rationale: Online courses provide an opportunity for flexible and adaptive nutrition education delivery. The unique environment and cultural diversity of the vast Pacific region requires nutrition education that incorporates aspects of the "place," such as commonly consumed foods and their societal significance. Existing curricular materials, geared towards Western foods and culture, do not meet this need.

Description: The OINC-HP was asynchronously delivered, and students completed weekly online modules (included lessons, discussions, and quizzes). Examples of integrating content included incorporating HP foods in lessons and specific videos and reading materials, weekly. This course meets two UH general education requirements: Biological Sciences and Hawaiian, Asian, and Pacific Issues. Students provided feedback in a midsemester survey.

Evaluation: Approximately $80 \%$ of students agreed that HP content integration was beneficial. Students commented that the course was relevant to living in HP. Discussions were identified as the most successful HP integration component because it allowed for the connection and application of concepts. A few students commented that the HP content was not relevant since they were not from the region.

Conclusions and Implications: Positive feedback on HP content integration implies that nutrition education can be successfully delivered with a place-based focus. Further investigation is needed on delivery methods of HP content to make it relevant for all.

Funding: NIFA, Association of Public and Land-grant Universities, Grant \#2011-68001-30335. 